\title{
Ein Vergleich der Lösse der Wischauer Senke (Mähren) mit den Lössen der angrenzenden Gebiete
}

\author{
Von Rudolf Musil und Karel Valoch, Mährisches Museum in Brünn (Brno)
}

Abstract: This paper deals with the loess layers in the western part of the Vyškov low-ground between Sokolnice and Ivanovice in Hana district in the area east of Brno in Moravia. During the Würm period three loess layers were formed here, being separated from each other by fossil soils. Loesses of Würm 3 are of a brownish colour, darker than the older loesses. The Interstadial Würm $2 / 3$ has the appearance of a brown loess soil, often with a foliaceous structure. The loess of Würm 2 is sometimes absent; this period is distinguished by strong periglacial phenomena. The Interstadial Würm $1 / 2$ is always developed as black earth, often doubled. The loess of Würm 1 forms in most cases the base of the brick pits, while the older Pleistocene sediments where found only in isolated cases.

The results obtained are in agreement with the observations made during our study of the loess in the neighbourhood of Brno and also with those made in the surrounding area, particularly in lower Austria.

Ré s u mé. Notre étude s'occupe des dépôts de loess dans l'Ouest de Vyškov entre Sokolnice et Jvanovice à l'Est de Brno en Moravie. Pendant le Würm trois couches de loess se sont formées, séparees l'une de l'autre par de sols fossiles. Les loess du Würm 3 sont de couleur brunâtre plus foncé que les loess plus anciens. L'interstade Würm 2/3 paraît comme une terre loessique brune, souvent avec une structure à feuillettes. Les loess du Würm 2 manquent quelque fois. Cette période est caracterisée par de forts phénomènes periglaciaires. L'interstade Würm 1/2 paraît toujours comme un tchernozem souvent redoublé. Les loess du Würm 1 forment pour la plupart la base des briqueteries; les sediments de Pleistocène plus ancients ne se trouvent que rarement.

Ces resultats correspondent aux connaissances acquises pendant l'étude des loess dans les environs de Brno et aussi à celles qu'on a acquises dans les regions voisines, surtout en Basse Autriche.

Das Gebiet der Wischauer Senke, welches der Gegenstand unserer Untersuchungen war, liegt östlich von Brünn (Mähren), ungefähr zwischen dem Steinitzer Wald und Ausläufern des Drahaner Höhenzuges. Das Gelände ist mäßig gewellt und unterscheidet sich dadurch von dem Gebiet in der Umgebung von Brünn. Das Landschaftsrelief beeinflußt auch den Zustand der Erhaltung der Lößdecken, die hier von relativ geringerer Mächtigkeit sind. Größtenteils sind bloß drei Lößdecken entwickelt, und nur ausnahmsweise sind ältere pleistozäne Sedimente erhalten.

Die vorgelegte Arbeit knüpft an das Studium pleistozäner Ablagerungen in der Umgebung von Brünn an (Musil, VAloch, NečEsaný 1955, Musil \& VAlOch 1955), und bezweckt die Feststellung charakteristischer durchgehender Merkmale auf diesem Gebiet. Es wurden alle dort befindlichen Lößgruben untersucht.

Eine eingehende Beschreibung der einzelnen Profile mit daraus folgenden Schlüssen befindet sich in unserer ursprünglichen Arbeit, die mit schematisierten Profilzeichnungen belegt ist (Musil \& VALOCH 1956).

Die Lösse der Wischauer Senke wurden zum guten Teil im Laufe des letzten Glazials gebildet. Im allgemeinen sind bloß drei Lößlagen entwickelt, die durch fossile Bodenbildung voneinander getrennt sind. Altere Ablagerungen sind nur vereinzelt vertreten. Schon beim Studium der Lösse aus der Umgebung von Brno war zu sehen, daß ältere Lösse desto weniger erhalten blieben, je flacher die Landschaft ist. Die Arbeiten in der Wischauer Senke haben diese Vermutung voll bestätigt.

Bei der Zusammenfassung der durchgehend erscheinenden Merkmale wurde erkannt, daß viele den bereits an den Lössen der Brünner Umgebung festgestellten, gegebenenfalls jenen durch andere Autoren aus andern Gebieten beschriebenen entsprechen. Es 
wird deshalb Aufgabe der folgenden Abschnitte sein, die in dieser Gegend erzielten Ergebnisse zusammenzufassen und $\mathrm{zu}$ vergleichen.

Die Lößdecke des Würm 3 bildet gelbbrauner Löß mit stärkerem Gehalt von ausgefälltem $\mathrm{CaCO}_{3}$, größtenteils in Form von kleinen Kalkkonkretionen bis zu Walnußgröße. Eine ähnliche Färbung der jüngsten Lösse wird auch aus andern Gebieten (Waagtal-Slowakei) erwähnt, und es hat den Anschein, daß dieses offenbar klimatisch bedingte Merkmal über ein größeres Areal verbreitet ist. Es ist sicher von Interesse, daß auch E. W. Guenther beim Studium der Lösse am Oberrhein über die Größe der KalkKonkretionen zu ähnlichen Ergebnissen gelangt. Obzwar es nicht angeht, so entfernte Gebiete zu vergleichen, ist die Erscheinung doch auffallend, daß auch dort in der obersten Lößschichte kleinere Kalkkonkretionen erscheinen, während die Konkretionen der älteren Lösse immer größer sind, so daß man nach Guenther schon nach der durchschnittlichen Größe der Kalkkonkretionen auf ihre Herkunft schließen kann (GuENTHER 1953). Auch die Autoren der vorliegenden Arbeit gelangten zu demselben Schluß. Der Löß dieser Periode zeigt in der Wischauer Senke eine typische prismatische Abspaltung, und nur sehr selten (Dědice, Letošov) wurden Zeichen einer Einwirkung des periglazialen Klimas beobachtet. Es kam also, wenigstens in diesem Gebiet, nur ausnahmsweise zur Bildung von Eiskeilen und Solifluktionsvorgängen.

Beim makroskopischen Studium gelang es nicht, die beschriebene Lößdecke in zwei Sichichten zu teilen, wie dies J. PeLíšEK (1954) auf Grund von Korngrößenanalysen tat. Nach Pelíšer enthält jeder Löß an der Basis am meisten Sand und am wenigsten Staub, während das Verhältnis an der Oberfläche der Decke umgekehrt ist. K. ŽEBere etc. (1955) in Předmostí und B. Klíma (1955) in Petřkovice teilen den jüngsten Löß nach den vorhandenen Solifluktions-Horizonten.

Der Horizont des Würm 2/3 war ebenso wie in der Umgebung von Brno in Form von braunen, lößartigen Erden entwickelt und wird nach Osten hin durch eine Schichte von bräunlicher Erde ersetzt, die sich vom Hangenden und Liegenden deutlich durch blättriges Gefüge abhebt. Auch in diesem Horizont treten periglaziale Erscheinungen nur ganz vereinzeilt auf.

Eine ähnliche Horizontbildung bräunlicher lößartiger Erden der betreffenden Periode wird nicht nur aus andern Gebieten der ČSR, sondern auch aus Niederösterreich beschrieben. Auch dort wurden keine Spuren einer Solifluktionstätigkeit festgestellt (Brandtner 1954). Die Autoren nehmen an, daß sich die Bildung des erwähnten Horizontes im Hinblick auf die klimatischen Verhältnisse des Stadials unter mäßig erhöhter Temperatur und bei ausgiebigeren Niederschlägen vollzog. Im Lößaufschluß von Tvarožná liegt unter dem beschriebenen Horizont stellenweise eine schwache rostbraune Erdschichte. Hier handelt es sich wahrscheinlich um eine gewisse Gliederung des letzten Interstadials, die BrANDTNER auch im westlichen Teil von Niederösterreich gefunden hat.

An manchen Stellen der Umgebung von Brno, z. B. in Rozdrojovice (MusiL 1955), wurde festgestellt, daß der pleistozäne Horizont des Würm 2/3 größtenteils zum holozänen B-Horizont umgewandelt war. Bloß an günstig gelegenen Stellen war eine schwache Schichte der unverwandelten braunen lößartigen Erde erhalten geblieben. Dieselbe Erscheinung beschreibt Fr. Brandtner (1954) von mehreren Orten in Niederösterreich. Ein analoger Fall kam in der Ziegelei von Topolany vor, wo der letzte WürmLöß durch den Bodenbildungsprozeß in einen holozänen B-Horizont verwandelt war. Nur an wenigen Stellen blieb er in geringer Mächtigkeit unverändert erhalten.

Bei den Lössen des zweiten Würm-Stadials kann man im allgemeinen dieselben charakteristischen Hauptmerkmale konstatieren, die schon bei früheren Studien festgestellt wurden. Die Lösse des Würm 2 sind überwiegend von geringer Mächtigkeit und fehlen oft ganz; periglaziale Erscheinungen sind stark entwickelt. Ahnliche Merkmale beschränken sich nicht nur auf dieses Gebiet, sondern wurden auch andernorts konstatiert 
(ŽEbera etc. 1955, ProžEk-Ložex 1954). Zum Unterschied vom hangenden Löß ist die beschriebene Schichte ockergelb gefärbt und kalkarm.

Periglaziale Erscheinungen treten in dieser Periode in verschiedenen Zeitabständen auf. Manchmal ist die interstadiale Schicht im Liegenden, in der sich lange Eiskeile und an der Oberfläche dünne Fließerdeschichten bilden, stark betroffen. Die Eiskeile sind mit humosen dunklen Erden offenbar aus der umgebenden Schwarzerde ausgefüllt, und die von der Solifluktion betroffenen Oberpartien sind durch dünne Schichten gebildet, deren Farbe zwischen Schwarz und Braun schwankt. Sie entstanden offenbar noch vor der Lößablagerung, in der ersten Hälfte des Stadials. Zwei weitere Solifluktionslagen, die nur im günstigsten Fall entwickelt sind, entstanden während der Lößablagerung, und zwar an ihrem Anfang und an ihrem Ende. Das bedeutet, daß das Klima etwas feuchter sein mußte als zur Zeit der stärksten Lößsedimentation, wo ausgesprochen kontinentales Klima herrschte. Chronologisch kann man also drei verschiedene Phasen der Bildung von Solifluktions-Horizonten unterscheiden.

Solifluktionserscheinungen können in der Wischauer Senke in viel größerem Ausmaß beobachtet werden als in der Umgebung von Brno. Die Lösse des beschriebenen Gebiets liegen meist auf Tegeln, und die Landschaft ist flacher, so daß das Wasser nicht so schnell abfließen konnte, wobei die Verdunstung bei kühlem Klima minimal war. Diese Umstände haben die Solifluktion sicher begünstigt.

Wie erwähnt, ist der Löß der betreffenden Periode relativ schwach entwickelt, was im Zusammenhang mit der gewaltigen Solifluktionstätigkeit auffällt. Die Solifluktion erforderte gewiß ein humideres, der Lößbildung weniger günstiges Klima. Die erhöhte Solifluktionstätigkeit selbst entfernte dann oft auch diese relativ schwache Lößschichte. Daher trifft man Profile an, wo über der Schwarzerde des Würm 1/2 ein solifluktionell geschichteter Horizont und über ihm ein wenig ausgeprägter Bodentypus mit dem letzten bräunlichen Löß liegt. Ja es kommt besonders in flacherem Gelände vor, daß die Lösse des Würm 2 nicht anwesend sind, so daß im Würm-Profil tatsächlich nur zwei typische Lößschichten (W 1 und W 3 ) vorhanden sind. Vielleicht hat gerade dieser Umstand manche Autoren veranlaßt, im letzten Würm-Glazial nur zwei Lösse zu unterscheiden.

Ganz anders als der Horizont des Würm 2/3 sind die Sedimente des ersten WürmInterstadials gebildet. Ahnlich wie in der Umgebung von Brno wiederholen sich hier meist verdoppelte Horizonte mit periglazialen Begleiterscheinungen, die im folgenden Stadial entstanden. Fast bei allen Profilen ist im Liegenden der interstadialen Schichtenfolge von Braun- und Schwarzerde eine Schichte rostbrauner bröckeliger Erde entwickelt, die prismatische Struktur hat und sich auffallend von den oberen humosen Lagen unterscheidet. Ihr Liegendes bildet meist ein Ca-Horizont von verschiedener Stärke durch Anhäufungen von kalkigen Pseudomyzelien oder größeren Kalkkonkretionen. Die rostbraune Schichte selbst enthält gewöhnlich kein ausgefälltes $\mathrm{CaCO}_{3}$. Jedesmal liegt unmittelbar über diesem Horizont eine Schwarzerdeschicht, die gewöhnlich mit schwarzem Erdreich ausgefüllte Eiskeile ins Liegende sendet. Über ihr befindet sich eine solifluktionell geschichtete Lage, die aus einzelnen teils dunkleren, teils brauneren dünnen Schichten besteht. Nur im günstigsten Falle, wie z. B. beim Aufschluß in Tvarožná, liegt über dieser Schwarzerde eine schwächere Lage helleren braunen Erdreichs und schließlich eine ganz dünne Schichte, die etwas brauner und humoser ist als das Liegende.

Es ist also klar, daß die Entwicklung der Schichten im Laufe dieses Interstadials viel gegliederter war, als es die Mehrzahl der Profile zeigt. In vielen Fällen wurden die oberen Partien offenbar durch Solifluktionstätigkeit entfernt.

Aufmerksamkeit verdient der Umstand, daß viele dieses Interstadial kennzeichnende Merkmale sich nicht nur in der Umgebung von Brno wiederholen, was ja wegen der Nähe des Gebiets und der gleichen klimatischen Verhältnisse begreiflich ist, sondern 
auch in Niederösterreich erscheinen, wie es Brandtner (1954) beschreibt. Die Entwicklung der Schichten entspricht im großen ganzen der Wischauer Senke. Auf liegenden rostbraunen Erden, die BrandTNer konsequent als selbständigen Bodentyp und nicht als B-Horizont der folgenden Schwarzerden einreiht, gelang es ihm an vielen Stellen eine ganz schwache Schichte typischen Lösses zu finden, die den Horizont von der hangenden Schwarzerde trennt. Aus diesem Grunde und auch deshalb, weil die Lößstruktur der rostbraunen Schichte sehr gut erhalten ist, reiht er sie nicht als B-Horizont ein, obwohl hier die Anhäufung von $\mathrm{Fe}_{2} \mathrm{O}_{3}$, manchmal auch von $\mathrm{Al}_{2} \mathrm{O}_{3}$, größer ist als im liegenden Löß. Einen typischen B-Horizont läßt er bloß für Riß-Würm-Sedimente gelten, als die Landschaft bewaldet war, doch nicht für das Würm 1/2, als nach seiner Ansicht die Steppe vorherrschte. Nach diesem Autor ist das Riß-Würm die letzte wahre Waldperiode und das letzte klimatische Optimum, dessen Höhe später nicht mehr erreicht wurde.

Beim Studium zur vorliegenden Arbeit konnte die dünne Trennungsschichte typischen Lösses kein einziges Mal festgestellt werden. Auch die anderen heimischen Forscher konnten sie, soweit bekannt, nie beobachten. Die rostbraune Schichte selbst hat größtenteils prismatischbröckelige Struktur und liegt immer unter der tiefsten Schwarzerde der betreffenden Periode.

J. Pelíšek (1954) reiht die rostbraune lößartige Erde in seiner Arbeit über das Quartär der östlichen Umgebung von Brno (Ziegelei unter Nová hora) als BraunerdeHorizont ein.

Die obere Schwarzerde der österreichischen Aufschlüsse ist durch zwei Oszillationen in drei Schichten geteilt, wo die äolische Lößablagerung überwog. Die untere Schichte enthält am meisten Humus und zeigt die dunkelste Färbung; nach oben zu vermindert sich nach und nach der Humusgehalt, und die Intensität der Färbung und die Schichte geht allmählich in hellen Löß über. FR. BrandtNer (1954) ist der Ansicht, daß die schwach humosen braunen Lößschichten bei ständiger leichter äolischer Sedimentation entstanden. Eine ähnliche Entwicklung der Schichten kann man auch bei manchen hier untersuchten Profilen verfolgen. Doch kam es meist zur Entfernung der oberen Partien der Schichtenfolge durch Solifluktionsvorgänge. Die Autoren der vorliegenden Arbeit sind jedoch der Ansicht, daß wohl nicht alle die Schwarzerde trennenden Zwischenschichten auf diese Weise entstanden sind. Es kamen sicherlich auch andere Einflüsse zur Geltung, und man muß daher jedes Profil selbständig im Hinblick auf seine besonderen Bedingungen beurteilen. So beobachtete z. B. R. LAIS (1954), daß die Schwarzerdeschichten der Ziegelei von Dolní Věstonice (Unter-Wisternitz) in einer Mulde liegen und daß die $Z_{w}$ ischenschichten nicht an Ort und Stelle durch Verwitterung des Lösses, sondern durch Zuschwemmung weniger verwitterten Materials aus der nahen Umgebung entstanden sind. Er dachte dabei nicht an die Wirkung der reichen Niederschläge, sondern eher an die allmähliche Einebnung der bestehenden Vertiefung.

Aus der Beschreibung der Schichten des gesamten Horizontes geht hervor, daß aus dieser Periode in optimalen Fällen mehrere Schichten erhalten bleiben konnten. Wenn man außerdem noch in Betracht zieht, daß die größere Mächtigkeit dieser Schichtenfolge nicht nur durch autochthone Sedimentbildung, sondern auch durch spätere Anhäufung infolge Anspülung oder Solifluktion (Bučovice, Mouř́nov) entstehen konnte, gelangt man zur Erkenntnis, daß die Sedimente des Würm 1/2 auch sekundär bedeutende Mächtigkeit erreichen konnten. Dieser Umstand kann leicht zur Vermutung Anlaß geben, daß sie dem letzten Interglazial angehören, obgleich es sich eigentlich um das erste WürmInterstadial handelt.

Auf Grund der bisherigen Kenntnisse kann man sagen, daß auf dem beschriebenen Gebiet im Laufe dieser Periode gleiche Bodentypen entstanden, die durch analoge klimatische Verhältnisse bedingt sind und vermutlich dem letzten Interglazial sehr ähnelten, 
von welchem sich das erste Interstadial selbst hauptsächlich durch die Länge seiner Dauer unterschied.

Die Lösse des Würm 1 liegen größtenteils schon auf älteren tertiären Unterlagen und sind gelb bis braungelb gefärbt. Sie enthalten hie und da Pseudomyzelien von $\mathrm{CaCO}_{3}$, allenfalls verstreute Kalkkonkretionen. Stellenweise kann man Wurzelröhrchen beobachten, die manchmal nur in bestimmten Lagen konzentriert sind und von dauerndem, ständig erneuertem Graswuchs zeugen. In der aktiven Ziegelei von Rousínov stieß die Untersuchung auf eine $10 \mathrm{~cm}$ starke dunklere Lage, die den Löß in zwei Partien teilte. Dieselbe Erscheinung beschrieben Fr. ProšEK \& V. LožEK (1954) von verschiedenen, ziemlich weit voneinander entfernten Orten. Die Autoren konnten sie auch bei der Untersuchung in Rozdrojovice feststellen (MusiL 1955). In der Lehmgrube von Rousínov wurde ferner im Löß des Würm 1 noch eine durchlaufende Lage blättrigen Gefüges angetroffen, die auch K. ŽEBera (1954) im Solifluktions-Horizont des Würm 1 fand und für einen wichtigen Leithorizont hält.

In vereinzelten Fällen sind noch ältere Riß-Würm-Sedimente vertreten. Doch sind sie nicht so mächtig wie in der Umgebung von Brno. $\mathrm{Da}$ sie nur vereinzelt und ungenügend entwickelt vorkommen, kann man keine allgemeineren Schlüsse ziehen. Doch handelt es sich um dieselben Ablagerungen, die Fr. Brandtner (1954) in Niederösterreich beschreibt und als Kremser Bodentypus bezeichnet. Altere, wahrscheinlich RißLösse sind nur ganz ausnahmsweise erhalten.

Die in der Wischauer Senke erzielten Erkenntnisse beweisen voll die Richtigkeit der Schlüsse sowohl unserer wie auch anderer Studien. Im letzten Glazial sind immer, falls es nicht durch örtliche Einflüsse verändert ist, drei Lösse erhalten. Ohne ihre Eigenschaften näher zu beschreiben, wollen wir nochmals kurz besonders auf die in Interstadialzeiten gebildeten Sedimente aufmerksam machen.

Die Ablagerungen des letzten Würm-Interstadials (W 2/3) weisen nur auf eine nur sehr schwache Erwärmung. Dagegen sind die Ablagerungen des ersten Interstadials (W 1/2) immer als Schwarzerden entwickelt, die manchmal verdoppelt sind. Ahnlich als Schwarzerden sind auch Ablagerungen des letzten Interglazials (R-W) entwickelt, allerdings in relativ größeren Mächtigkeiten. Die interstadialen Sedimente unterscheiden sich von den interglazialen bloß dadurch, daß sie immer in geringerer Mächtigkeit hervortreten. Wir vermuten daher, daß beide Warmzeiten nicht einmal so durch die Intensität, als durch die Dauer des Klimaeinwirkens unterschieden sind. Es besteht bei uns kein Grund, den kürzeren Zeitabschnitt des Würm 1/2 als Interglazial und die tieferliegenden Sedimente einer viel längeren Warmzeit als ein vorangehendes Interstadial zu bezeichnen. Diese Auffassung wird auch durch andere Umstände, z. B. durch starke, am Ende des Interglazials erscheinende Abtragung, bekräftigt.

Zusammenfassend kann man sagen, daß im Untersuchungsgebiet meist drei Lößdecken entwickelt sind, die den einzelnen Würmstadialen angehören. Die Autoren können mit der $\mathrm{ab}$ und zu geäußerten Ansicht nicht übereinstimmen, es sei während des letzten Glazials allgemein nur eine Lößdecke angeweht worden. Diese Behauptung ist wohl durch die Tatsache verschuldet, daß die Ablagerungen dieser Periode in bestimmten Gebieten ungenügend entwickelt und erhalten sind oder daß wenig typische Profile unrichtig gedeutet wurden. Ebenfalls kann man nicht der Gleichstellung des Würm 1/2 mit dem Interglazial zustimmen. Die Untersuchung der Lösse des Wischauer Gebiets und der Umgebung von Brünn bietet eine gute Stütze für Soergen's Gliederung des letzten Glazials in drei Stadiale, denen die drei Lößdecken entsprechen.

Vor der Drucklegung dieses Artikels erschien eine Reihe von Arbeiten, die sich mit ähnlichen Lößfragen beschäftigen, von denen besonders die umfangreiche Arbeit Fr. BRandTNER's (Eiszeitalter und Gegenwart 7, 1956) für unsere Vergleichsstudien von Wichtigkeit ist, auf die aber leider nicht mehr Bezug genommen werden konnte. 


\section{Liter a tur:}

Brandtner, Fr.: Über die relative Chronologie des jüngeren Pleistozäns Niederösterreichs. Archaeologia Austriaca 5, S. 101113. 1950. - - Jungpleistozäner Löß und fossile Böden in Niederösterreich. - Eiszeitalter und Gegenwart 4/5, S. 49-82, 1954.

Fink, J.: Die fossilen Böden im österreichischen Löß. - Quartär 6, S. 85-108, 1954.

FreIsING, H.: Gibt es in Hessen drei Würmlösse? - Jber. u. Mitt. oberrh. geol. Ver. 35, S. 54-66, 1954.

Guenther, E. W.: Feinstratigraphische Untersuchung eines Lößprofils von Riegel am Kaiserstuhl. - N. Jb. Geol. Paläont. 1953, S. 369-385.

KLímA, B.: Výsledky archeologického výzkumu na tàbořišti lovců mamutů v Petřkovicích, okr. Ostrava, v roce 1952 a 1953. - Acta Musei Silesiae 4, S. 1-35, 1955.

LAIS, R.: Über den jüngeren Löß in Niederösterreich, Mähren und Böhmen. - Ber. naturforsch. Ges. Freiburg i. Br. 41, S. 119-178, 1951. - - Uber den Löß von Unter-Wisternitz (Mähren). - Palaeohistoria 2, S. 135-170, 1954.

Musil, R.: Geologická situace na paleolitickém nalezišti v Rozdrojovicích u Brna. - Acta Musei Moraviae 40, sc. nat. S.5-37, 1955.

Musil, R. \& VALOCH, K.: Uber die Erforschung der Lösse in der Umgebung von Brünn (Brno) in Mähren. - Eiszeitalter und Gegenwart 6, S. 148-151, 1955. - - Spraše Vyškovského úvalu. - Acta Acad. Scient. Čechoslov., Basis Brunensis 28. H. 6, 263-307, 1956.

Musil, R., Valoch, K., NećEsaný, Vl.: Pleistocenní sedimenty okolí Brna. - Anthropozoikum 4, S. 107-167. Praha 1955.

Pelíšex, J.: Cernozemě pohřbené (diluviální) ve spraších od Vyškova. - Sborník Přírodov. klubu v Brně, sep. 1-5, 1938. - - Kvartér východního okolí Brna. - Anthropozoikum 3, S. 7-28. Praha 1954.

PRošEK, Fr., LožEK, V.: Stratigrafické otázky československého paleolitu. - Památky archeologické 45, S. 35-74, 1954. - - Sprašový profil v Bance u Pieštan (záp. Slovensko). Anthropozoikum 3, S. 301-323. Praha 1954.

Schönhals, E.: Fossile gleiartige Böden des Pleistozäns im Usinger Becken und am Rand des Vogelbergs. - Notizbl. hess. Landesamt f. Bodenforsch. Wiesbaden, N. F. 6, S. 160 bis 183, 1951. - - Uber fossile Böden im nichtvereisten Gebiet. - Eiszeitalter und Gegenwart 1, S. 109-130, 1951.

ŽEbera, K.: Výsledky výzkumu kvartérních sedimentů v Předmostí u Přerova na Moravě za rok 1952. - Anthropozoikum 3, S. 139-170, 1954.

Žebera, K., Ložek, V., Kneblová, Vl., Fejfar, O., MazáleK, M.: Zpráva o druhé etapě geologického výzkumu kvartéru v Předmostí u Přerova na Moravě. - Anthropozoikum 4, S. 291-362. Praha 1955.

Anschrift der Verf.: Dr. Rudolf Musil und Karel Valoch, Moravské Museum v Brné (Brünn), Tschechoslowakei. 